\title{
Hanle signatures of the coronal magnetic field in the linear polarization of the hydrogen $\mathrm{L} \alpha$ line
}

\author{
M. Derouich ${ }^{1, \star}$, F. Auchère ${ }^{1}$, J. C. Vial ${ }^{1}$, and M. Zhang ${ }^{2}$ \\ 1 Institut d'Astrophysique Spatiale, CNRS-Université Paris-Sud 11, 91405 Orsay Cedex, France \\ e-mail: moncef.derouich@ias.u-psud.fr \\ 2 National Astronomical Observatory, Chinese Academy of Sciences, Beijing 100012, PR China
}

Received 3 July 2009 / Accepted 21 October 2009

\begin{abstract}
Aims. This paper is dedicated to the assessment of the validity of future coronal spectro-polarimetric observations and to prepare their interpretation in terms of the magnetic field vector.

Methods. We assume that the polarization of the hydrogen coronal $\mathrm{L} \alpha$ line is due to anisotropic scattering of an incident chromospheric radiation field. The anisotropy is due to geometrical effects but also to the inhomogeneities of the chromospheric regions which we model by using Carrington maps of the $\mathrm{L} \alpha$. Because the corona is optically thin, we fully consider the effects of the integration over the line-of-sight (LOS). As a modeling case, we include a dipolar magnetic topology perturbed by a non-dipolar magnetic structure arising from a prominence current sheet in the corona. The spatial variation of the hydrogen density and the temperature is taken into account. We determine the incident radiation field developed on the tensorial basis at each point along the LOS. Then, we calculate the local emissivity vector to obtain integrated Stokes parameters with and without coronal magnetic field.

Results. We show that the Hanle effect is an interesting technique for interpreting the scattering polarization of the $\mathrm{L} \alpha \lambda 1216$ line in order to diagnose the coronal magnetic field. The difference between the calculated polarization and the zero magnetic field polarization gives us an estimation of the needed polarimetric sensitivity in future polarization observations. We also obtain useful indications about the optimal observational strategy.

Conclusions. Quantitative interpretation of the Hanle effect on the scattering linear polarization of $\mathrm{L} \alpha$ line can be a crucial source of information about the coronal magnetic field at a height over the limb $h<0.7 R_{\odot}$. Therefore, one needs the development of spatial instrumentation to observe this line.
\end{abstract}

Key words. line: formation - Sun: corona - Sun: UV radiation - scattering

\section{Introduction}

One of the most powerful tools for the diagnostics of magnetic fields in the Sun is the interpretation of polarimetric observations (e.g. the monograph by Landi Degl'Innocenti \& Landolfi 2004; and the recent review by Trujillo Bueno 2009). However, these diagnostics are mostly concerned with the fields at the photospheric and chromospheric levels. The coronal magnetic field presents more intrinsic difficulties to measure and interpret. This is especially true for the case of the UV coronal lines. Only rather recently, Raouafi et al. (2002) performed the first measurement and interpretation of the linear polarization of a UV line (O VI $\lambda 1032$ line) polarized under anisotropic scattering by the underlying solar radiation field. In addition, Manso Sainz \& Trujillo Bueno (2009) proposed a polarizing mechanism showing the adequate sensitivity of other coronal UV lines to the direction of the magnetic field. These successful works suggest that new UV polarimeters with high sensitivity associated with theoretical and numerical modeling obtained with a high degree of realism are a fundamental step to be performed in order to extract information on the coronal plasmas. In this context, the

^ Present address: Colorado Research Associates Division, NorthWest Research Associates, Inc., 3380 Mitchell Ln., Boulder, CO 80301, USA.
Hanle effect on the L $\alpha$ polarization constitutes an excellent opportunity which merits to be exploited.

The scattering polarization of the coronal $\mathrm{L} \alpha$ line of neutral hydrogen, which we are revisiting in this paper, has been computed by Bommier \& Sahal-Bréchot (1982) and by Trujillo Bueno et al. (2005). These authors, however, neglected the effects of the integration over the line-of-sight (LOS) by considering a local position of the scattering hydrogen atom. Since the corona is optically thin, the LOS integration problem has to be solved. Fineschi et al. (1992) treated the case of the L $\alpha$ line polarization and took into account the LOS integration. However, Fineschi et al. considered the effect of a deterministic magnetic field vector having a direction and strength independent of the position of the scattering volume. They also treated the case of a random magnetic field.

To improve upon these previous works, we take into account the variation of the direction and the strength of the magnetic field for each scattering event along the LOS. The calculation of the polarization generated by scattering depends strongly on the level of anisotropy of the incident radiation, which in turn depends strongly on the geometry of the scattering process and the brightness variation of the chromospheric regions. In order to accurately compute the degree of the anisotropy at each scattering position, we use Carrington maps of the chromospheric incident radiation of the L $\alpha$ line obtained by Auchère (2005). In addition, 
the coronal density of the scattering atoms and the local temperature are included according to a quiet coronal model (Cranmer et al. 1999). We perform a comparison of the L $\alpha$ linear polarization in the zero-field reference case with the amplitude corresponding to the polarization in the presence of a magnetic field. In our forward modeling, we adopt a dipolar magnetic distribution as a first step and then we add a magnetic field associated to an equatorial current sheet.

The paper is organized as follows. We describe the theoretical background and formulate the problem in Sect. 2. Section 3 deals with the calculations of the Hanle effect without integration over the LOS in order to compare with known results. The generalization of these calculations to integrate over the LOS and the discussion of the possibility of obtaining a coronal magnetic field through polarization measurements are presented in Sect. 4. The technique that could be used to measure the scattering polarization of the $\mathrm{L} \alpha \mathrm{D}_{2}$ line is given in Sect. 5; in particular we show how the linear scattering polarization could be measured using a L $\alpha$ disk imager and coronagraph called LYOT (LYman Orbiting Telescope). In Sect. 6 we summarize our conclusions.

\section{Formulation of the problem}

\subsection{Hanle effect}

The term Hanle effect represents the ways in which the scattering polarization can be modified by weak magnetic fields. The well-known Zeeman effect and the Hanle effect are complementary because they respond to magnetic fields in very different parameter regimes. The Zeeman effect depends on the ratio between the Zeeman splitting and the Doppler line width. The Hanle effect though depends on the ratio between the Zeeman splitting and the inverse life time of the atomic levels involved in the process of the formation of the polarized line. For the permitted UV lines, the Zeeman effect is of limited interest for the determination of the magnetic fields in the quiet corona. This is because the ratio between the Zeeman splitting and the Doppler width is small due to the weakness of the magnetic field and the high Doppler width in such hot coronal plasmas. On the contrary, the measurement and physical interpretation of the scattering polarization of the UV lines are a very efficient diagnostic tool for determining the coronal magnetic field through its Hanle effect.

\subsection{Atomic linear polarization}

The possibility of the creation of a linear polarization by anisotropic scattering can be only explained correctly in the framework of the quantum-mechanical scattering theory. In fact, the intrinsic capacity of a line to be polarized is intimately linked to subtle quantum behaviors pertaining to the atomic levels involved in the transition. Let us denote by $m_{J}$ the projection of the orbital angular momentum $J$ of the hydrogen atom; $m_{J}$ takes the values $-J, J+1, \ldots, J$. The term "atomic linear polarization" in a $J$-level consists in (e.g. Cohen-Tannoudji \& Kastler 1966; Omont 1977; Sahal-Bréchot 1977; Blum 1981):

- an unbalance of the populations of the Zeeman sub-levels having different absolute values $\left|m_{J}\right|$;

- a presence of interferences between these Zeeman sub-levels.

This means that by definition, only levels having $J>1 / 2$ can be linearly polarized.

\subsection{Linear scattering polarization in the $L \alpha$ line}

The so-called scattering polarization is simply the observational manifestation of the atomic polarization. The Hanle effect is nothing but a perturbation of the atomic polarization by a magnetic field. The Hanle signatures in the spectrum of the linear polarization are a variation of the polarization degree and a rotation of the polarization plane. These Hanle signatures can be used to retrieve information on coronal magnetic fields. The two components $\mathrm{D}_{1}$ and $\mathrm{D}_{2}$ of the $\mathrm{L} \alpha$ connect the hydrogen ground state ${ }^{2} \mathrm{~S}_{J=1 / 2}$ to the electronic excited states ${ }^{2} \mathrm{P}_{J=1 / 2}$ and ${ }^{2} \mathrm{P}_{J=3 / 2}$, respectively. The upper level ${ }^{2} \mathrm{P}_{3 / 2}$ of the $\mathrm{D}_{2}$ line can be polarized due to the difference of the populations between the Zeeman sub-levels with $\left|m_{J}\right|=1 / 2$ and $\left|m_{J}\right|=3 / 2$. However, the states ${ }^{2} \mathrm{~S}_{1 / 2}$ and ${ }^{2} \mathrm{P}_{1 / 2}$ cannot be polarized since $\left|m_{J}\right|$ is necessarily $1 / 2$ implying that no difference of population inside these states can be generated by anisotropic scattering. Consequently, the $\mathrm{D}_{1}$ line is not linearly polarizable.

It is useful to keep in mind that in the description of the emitting hydrogen atom, we neglect the contribution of the hyperfine structure (HFS). For instance if the HFS is not neglected, the level $J=1 / 2$ of the ground state ${ }^{2} \mathrm{~S}_{1 / 2}$ is split into hyperfine levels $F=0$ and $F=1$ due to coupling with the nuclear spin of the hydrogen $I=1 / 2$. The hyperfine level $F=1$ can be linearly polarized ${ }^{1}$, which means that the $\mathrm{D}_{1}$ line can be polarized and the polarization of the $\mathrm{D}_{2}$ line can be affected. As previously suggested by Bommier \& Sahal-Bréchot (1982), we neglect the effect of the HFS in the process of formation of $\mathrm{L} \alpha$ line.

\subsection{Expression of the Stokes parameters}

The emission of the $\mathrm{L} \alpha \lambda 1216$ line in the solar corona has been discovered by Gabriel et al. (1971). They concluded that in most coronal structures the process responsible for the formation of the $\mathrm{L} \alpha$ line is the photo-excitation by underlying radiation. The creation of population imbalances and the quantum interferences in the ${ }^{2} \mathrm{P}_{3 / 2}$ and thus the existence of the scattering polarization in the $\mathrm{D}_{2} \mathrm{~L} \alpha$ line are caused by the photo-excitation of coronal neutral hydrogen by anisotropic chromospheric radiation (see Fig. 1).

The components of the incident radiation field at a frequency $v_{0}$ are usually denoted by $\bar{J}_{q}^{k}\left(v_{0}\right)$ where $k$ is the tensorial order and $q$ represents the coherences in the tensorial basis $(-k \leq q \leq k$ ); the order $k$ can be equal to 0 (with $q=0$ ) or 2 (with $q=0, \pm 1, \pm 2$ ). This radiation field with six components constitutes a generalization of the unpolarized light field where only the quantity $\bar{J}_{0}^{0}\left(v_{0}\right)$ is considered. In fact, $\bar{J}_{0}^{0}\left(v_{0}\right)$ is proportional to the intensity of the radiation.

If the incident radiation is no longer anisotropic, the components $\bar{J}_{q}^{k=2}\left(v_{0}\right)$ become zero, which means that no linear polarization can be created as a result of scattering processes. Regardless of the anisotropy of the incident radiation, the radiation component associated with the circular polarization usually denoted by $\bar{J}_{q}^{k=1}$ is negligible. This means that no odd order $k$ can be created inside the scattering hydrogen atom. As a result, the Stokes $V$ of the scattered radiation is zero.

We denote by $\zeta$ the angle between the direction of the incident light MP and the local vertical through the scattering center OP. The incident radiation comes from a chromospheric spherical cap limited by an angle $\zeta_{\max }$ corresponding to the tangent

\footnotetext{
1 In other words, population imbalances and quantum interferences between the sub-levels having $\left|m_{F}\right|=1$ and $\left|m_{F}\right|=0$ can be created due to the scattering of anisotropic light. The same is true for the hyperfine levels of the upper states ${ }^{2} \mathrm{P}_{3 / 2}$ and ${ }^{2} \mathrm{P}_{1 / 2}$.
} 


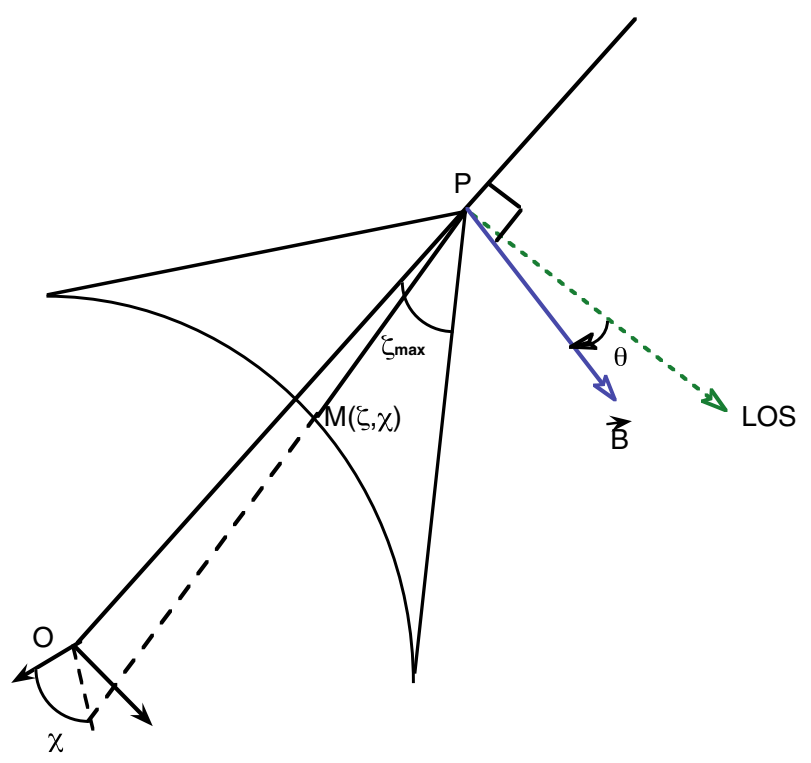

Fig. 1. Geometry of the scattering of chromospheric $\mathrm{L} \alpha$ photons by residual coronal neutral hydrogen. The anisotropy of the incident light is due to geometrical effects but also to the inhomogeneities of the chromospheric regions.

to the solar limb (see Fig. 1). $\chi$ is the azimuth angle around the normal with respect to an arbitrary reference. Note that $0 \leq \zeta$ $\leq \zeta_{\max }$ and $0 \leq \chi \leq 2 \pi$. When the distance from the solar surface increases, the anisotropy of the light becomes larger and the polarization degree increases. The maximum of polarization is reached when the radiation is purely directive, i.e. the spherical cap is seen by the scattering hydrogen atom as a point. It is useful to notice that if the chromosphere is assumed to be uniform the radiation has a cylindrical symmetry around its preferred direction, implying that the coherence components with $q \neq 0$ are zero. In fact, $\bar{J}_{q= \pm 1}^{k=2}\left(v_{0}\right)$ and $\bar{J}_{q= \pm 2}^{k=2}\left(v_{0}\right)$ components quantify the breaking of the cylindrical symmetry around the axis of quantification which is here the local vertical.

In the framework of the two level approximation, where only the upper level is polarized, the statistical equilibrium equations are solved analytically. The upper level density matrix elements are simply proportional to the incident radiation elements $\bar{J}_{q}^{k}$. The emissivity vector is then expressed as a function of the incident radiation field. Consequently, we do not explicitly calculate the density matrix elements, but instead we determine the incident radiation tensor at each scattering position along the line of sight. For an unmagnetized atmosphere, in an arbitrary reference, the emissivity vector can be written as (e.g. Landi Degl'Innocenti \& Landolfi 2004):

$\epsilon_{j}(\Omega)=n_{\mathrm{H}} \frac{h v_{0} B_{J_{l} J_{u}}}{4 \pi} \sum_{k, q} W_{k}\left(J_{l}, J_{u}\right) \mathcal{T}_{q}^{k}(j, \Omega)(-1)^{q} \bar{J}_{-q}^{k}\left(v_{0}\right)$

where $\Omega$ is the solid angle giving the direction of the LOS, $n_{\mathrm{H}}$ is the local number density of scattering hydrogen atoms, $h$ is the Planck constant, and $B_{J_{l}, J_{u}}$ is the Einstein coefficient for absorption. We recall that $\mathcal{T}_{q}^{k}(j, \Omega)$ is the spherical tensor for polarimetry which contains the angular distribution of the emitted radiation, and $j$ is the index of the Stokes parameter $(j=0,1,2$, and 3 for the Stokes $I, Q, U$, and $V$, respectively).

In order to determine the magnetic field one has to include its Hanle effect on the polarization of the $\mathrm{L} \alpha$ light, then, for a given magnetic field vector $\boldsymbol{B}, \epsilon_{j}(\Omega)$ becomes (e.g. Landi Degl'Innocenti \& Landolfi 2004):

$$
\begin{aligned}
\epsilon_{j}(\Omega, \boldsymbol{B})= & n_{\mathrm{H}} \frac{h v_{0} B_{J_{l} J_{u}}}{4 \pi} \\
& \times \sum_{k, q} W_{k}\left(J_{l}, J_{u}\right) \mathcal{T}_{q}^{k}(j, \Omega)(-1)^{q} \bar{J}_{-q}^{k}\left(v_{0}\right) \frac{1}{1+\mathrm{i} q H_{u}} .
\end{aligned}
$$

This expression of $\epsilon_{j}(\Omega, \boldsymbol{B})$ is correct only in a reference system having the quantization $z$-axis in the magnetic field direction. $H_{u}$ is the so-called reduced magnetic field strength, associated to the level ${ }^{2} \mathrm{P}_{3 / 2}$, given by:

$H_{u}=\frac{0.879 g_{u} B}{A_{J_{u} J_{l}}}$

where the Einstein coefficient for spontaneous emission $A_{J_{u} J_{l}}$ is given in $\left[10^{7} \mathrm{~s}^{-1}\right], g_{u}=4 / 3$ is the Landé factor of the level ${ }^{2} \mathrm{P}_{3 / 2}$ and the magnetic field strength $B$ is given in Gauss. $H_{u}=1$ corresponds to the magnetic field strength $B=53$ Gauss around which one may expect a noticeable change in the scattering polarization of $\mathrm{L} \alpha$ with respect to the unmagnetized reference case. The quantity $W_{k}\left(J_{l}, J_{u}\right)$ was first introduced by Landi Degl'Innocenti (1984) and depends only on the quantum numbers of the lower and upper levels $\left(J_{l}\right.$ and $\left.J_{u}\right)$ involved in the transition. For $k=2, W_{2}\left(J_{l}, J_{u}\right)$ can be seen as the efficiency of creation of the linear polarization in the scattering processes. That is why the $W_{2}\left(J_{l}=1 / 2, J_{u}=1 / 2\right)=0$ for the $\mathrm{D}_{1}$ line, which is not polarizable, but $W_{2}\left(J_{l}=1 / 2, J_{u}=3 / 2\right)=1 / 2$ for the polarizable $\mathrm{D}_{2}$ line.

\section{Hanle effect without integration over the LOS}

We developed a numerical code allowing for the calculation of the theoretical polarization taking into account the effects of the LOS. In order to validate the code, we considered typical cases of a horizontal magnetic field having different azimuth angles $\theta$ (angles between the magnetic field vector and the LOS). LOS integrations are avoided in order to be able to compare our results with well known Hanle effect results. We retrieve the Hanle behaviors typically encountered in the literature, for instance:

- when the magnetic field is zero or very small or oriented along the symmetry axis of the radiation field, the polarization is not affected;

- when the field increases until reaching the critical value corresponding to $H_{u}=1$, the polarization decreases rapidly. Moreover, for a very large $H_{u}$ (i.e. very large magnetic field strength) we obtain an asymptotical curve of polarization $p[B \rightarrow \infty]$ which depends only on the value of $\theta$ but not on the magnetic field strength. The asymptotic value of $p[B \rightarrow \infty]$ divided by $p[B=0]$ equals $1 / 5$ when the distribution of the magnetic field is isotropic ${ }^{2}$ and $1 / 4$ when the field has a cylindrical symmetry (i.e. horizontal magnetic field with random azimuth);

- no rotation of the plane of the polarization in the case of a highly symmetric distribution (e.g. isotropic or cylindrical) because the contributions of opposite magnetic polarities tend to cancel out;

2 The case of isotropic field distribution is encountered in the photosphere of the Sun (second solar spectrum) where the magnetic geometries are unresolved within the spatiotemporal resolution of the current observational capabilities. 


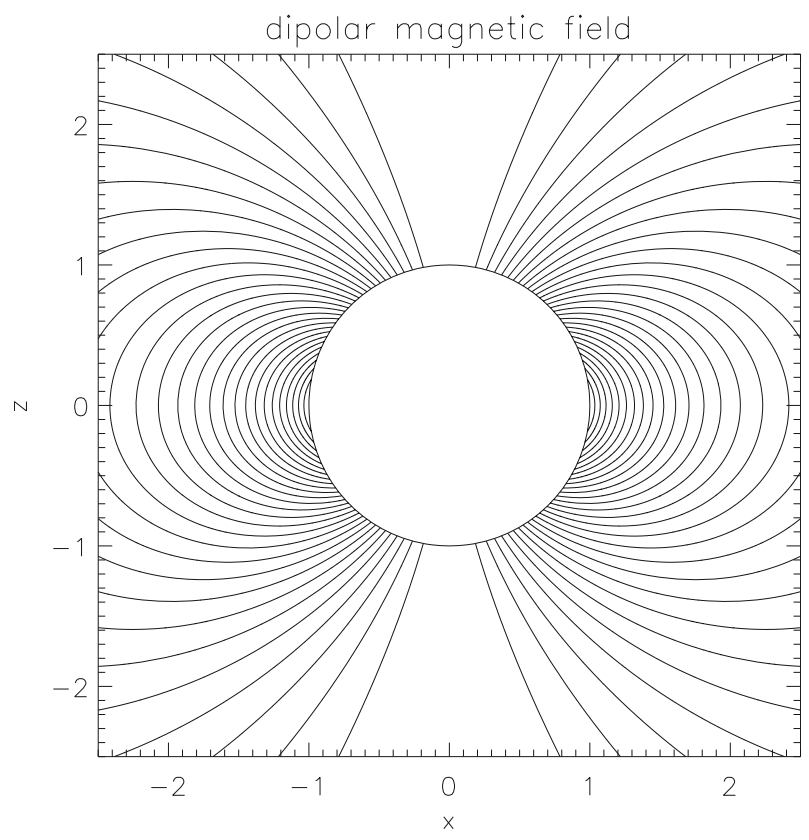

Fig. 2. A purely dipolar magnetic field structure presented in the plane $(x, z)$ in units of the solar radius $R_{\odot}$. We use a system of orthogonal Cartesian coordinates $(x, y, z)$ with the origin at the Sun center and the $z$-axis pointing toward the north solar pole.

- we find that a meridian magnetic vector (i.e. horizontal with $\theta=\frac{\pi}{2}$ ) presents a depolarizing effect without rotation of the polarization direction.

\section{Hanle effect integrated over the LOS}

The corona being an optically thin medium for the $\mathrm{L} \alpha$ line, it is then necessary to consider the effects of the integration over the LOS. We adopt the analytical magnetic field model proposed by Fong et al. (2002) and Low et al. (2003). It is a sum of two terms: a purely dipolar term and a term corresponding to the magnetic field of a current sheet structure. The model is axisymmetric and the prominence is treated as a cold plasma sheet forming a flat ring around the Sun. We take into consideration that the current is in the equator and that it represents a prominence sheet extending from $r=R_{\odot}$ to $r=\sqrt{4 / 5} R_{\odot}$. In the analytical expression of the magnetic field the contribution of the current sheet, relative to the dipolar background field is controlled by a constant ratio $\gamma$ (see Eq. (12) of Low et al. 2003).

\subsection{Purely dipolar magnetic field: $\gamma=0$}

As a first step, we avoid the effect of the term associated to the current sheet by taking $\gamma=0$. Figure 2 represents the dipolar term of the magnetic field. This configuration represents a typical coronal magnetic field of 15 to 20 Gauss close to the base of the corona. The Hanle effect of the dipolar magnetic field depends on the angle $\phi$ between the axis of symmetry of the incident light ${ }^{3}$ and the axis of symmetry of the magnetic structure. It also depends on the height above the solar surface $h$ mainly because the magnetic field strength decreases and the anisotropy

\footnotetext{
${ }^{3}$ Rigorously speaking, this is only the axis of symmetry of the spherical cap where the chromospheric radiation is uniform. The fact that the incident radiation is inhomogeneous implies that this symmetry around the preferred axis of radiation is broken.
}

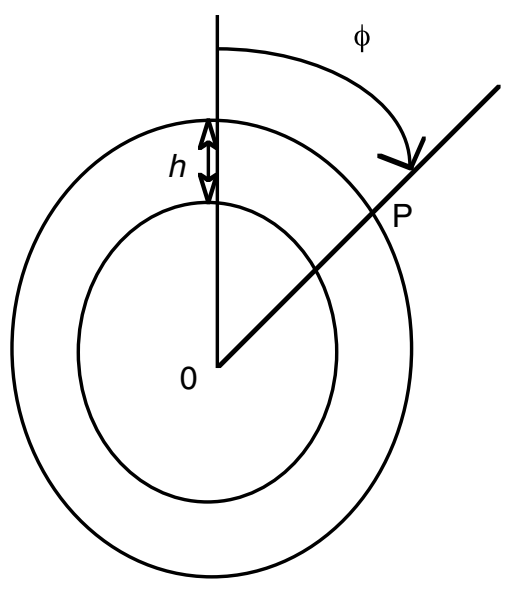

Fig. 3. The polarization at each position $\mathrm{P}$ of the scattering event depends on the height over the limb $h$ and the angle $\phi$ between the preferred axis of the radiation and the symmetry axis of the magnetic structure. The LOS is perpendicular to the plane of the figure.

of the incident light increases. The parameters $\phi$ and $h$ are represented in Fig. 3.

In theory, the expression of the emissivity vector is valid regardless of the location of the scattering atom. However, the integration over the LOS must take into account the inhomogeneities of the solar conditions like the variation of the hydrogen density, the temperature, the magnetic field and the variation of the incident radiation field. The density of neutral hydrogen and the temperature are assumed to be a function of the radial distance $r$ and the latitude (see Cranmer et al. 1999, for details). In order to model the inhomogeneities of the chromospheric intensity, we use the Carrington maps of the L $\alpha$ chromospheric line built by Auchère (2005). In the optically thin limit, the integrated Stokes parameters of the scattered radiation reduce to a volume integration over the LOS:

$\mathcal{E}_{j}(\Omega)=\int_{\mathrm{LOS}} \epsilon_{j}(\Omega) \mathrm{d} l$

then the polarization degree is

$p=\frac{\sqrt{\mathcal{E}_{1}^{2}+\mathcal{E}_{2}^{2}}}{\mathcal{E}_{0}}$

and the rotation of the direction of the polarization $\alpha_{0}$ is given by:

$\operatorname{tg}\left(2 \alpha_{0}\right)=\frac{\mathcal{E}_{2}}{\mathcal{E}_{1}}$

Figure 4 shows the variation of the linear polarization with the inclination $\phi$ for two different magnetic structures corresponding to two heights above the solar surface. Note that the ratio $p[B] / p[B=0]$ obtained for the height $h=0.3 R_{\odot}$ is smaller than the one corresponding to $h=0.5 R_{\odot}$ since the magnetic field decreases with $h$. Furthermore, as shown in Fig. 5, a notable Hanle rotation of about $10^{\circ}$ is obtained for $h=0.3 R_{\odot}$ and for $h=0.5 R_{\odot}$. Both Hanle signatures on the $\mathrm{L} \alpha$ line, i.e. depolarization and rotation (see Figs. 4 and 5), are clearly sizable for $\phi>40^{\circ}$. This important result suggests that in order to measure a dipolar magnetic field by its Hanle effect one should observe regions rather far away from the pole. 
M. Derouich et al.: Hanle effect in the coronal L $\alpha$ line

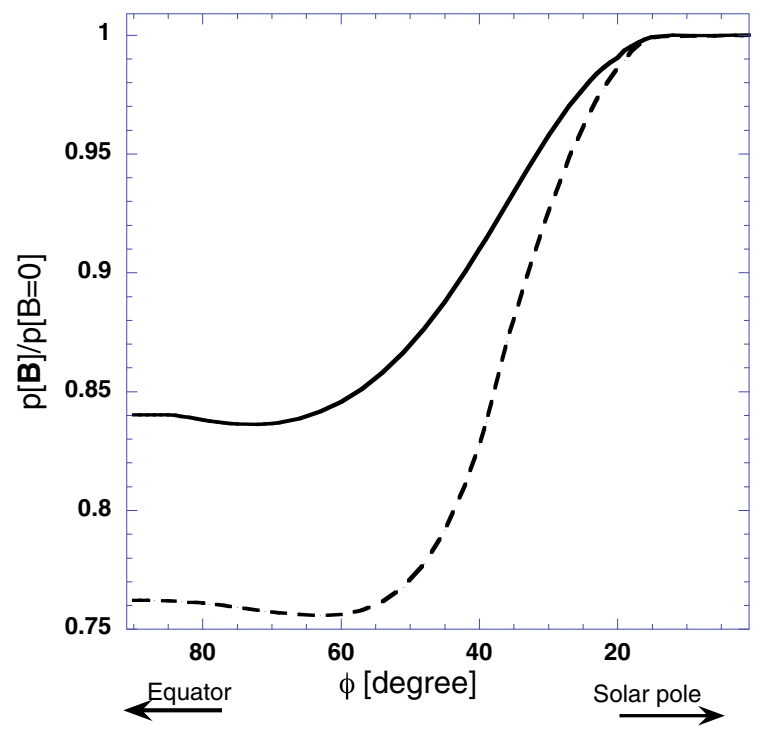

Fig. 4. Linear polarization degree obtained for a dipolar magnetic field divided by zero-field polarization versus the angle $\phi$. Full lines represent the polarization at $h=0.5 R_{\odot}$ and dashed lines represent the polarization at $h=0.3 R_{\odot}$

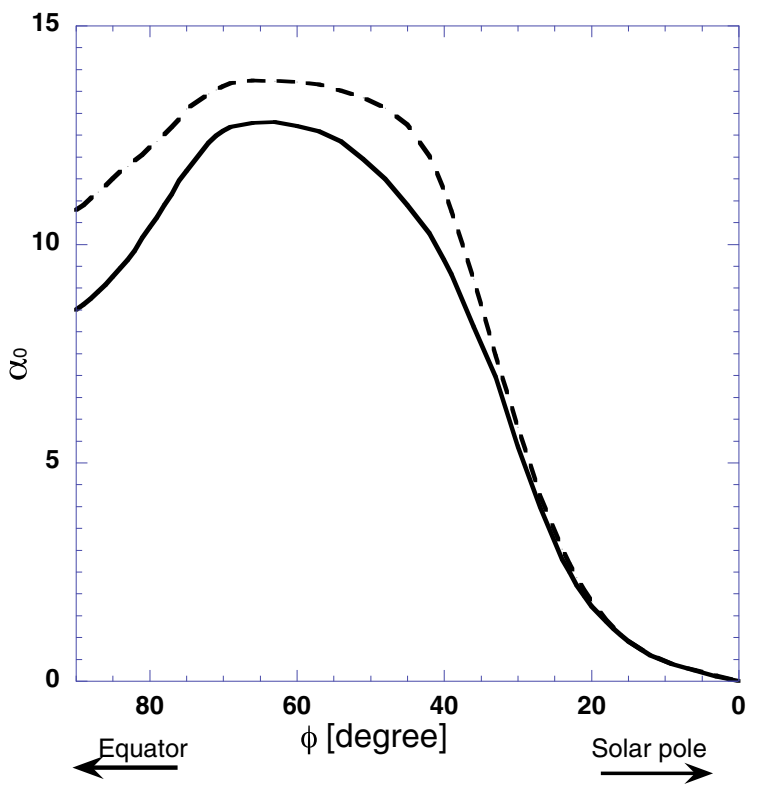

Fig. 5. Rotation angle obtained after integration over the LOS versus the inclination $\phi$ at $h=0.3 R_{\odot}$ and $h=0.5 R_{\odot}$. Full lines represent the rotation at $h=0.5 R_{\odot}$ and dashed lines represent the rotation at $h=0.3 R_{\odot}$.

\subsection{Perturbed dipolar field: $\gamma \neq 0$}

To the dipolar part of the magnetic field we now add the contribution resulting from an equatorial current sheet. We adopt a ratio $\gamma=0.25$ between the current sheet and the dipolar background field. In order to highlight the Hanle effect of the equatorial current sheet, we calculate the degree of polarization in a position located at $\phi=80^{\circ}$, and we vary the height above the limb $h$ (see Fig. 6). Figure 7 shows the difference between the linear polarization in the zero-magnetic field case and the one in the presence of the magnetic field, $\Delta p=\left|p_{B=0}-p_{B \neq 0}\right|$. A polarimetric sensitivity smaller than $\Delta p$ is needed in order to apply the Hanle effect as a technique of magnetic field investigations.

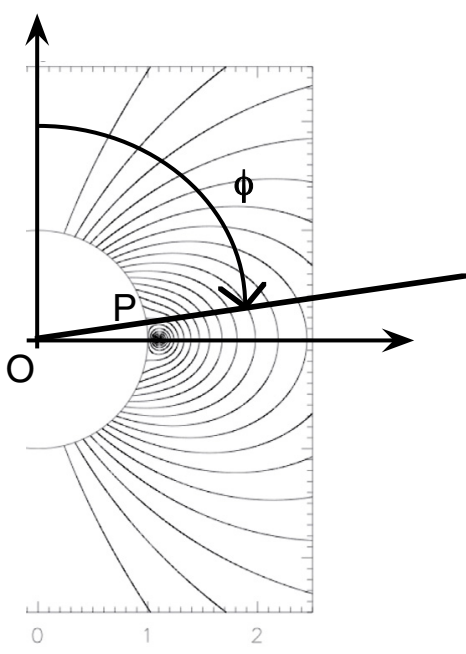

Fig. 6. Perturbation of the lines of the dipolar magnetic field due to an equatorial current sheet. The calculations of the polarization given in Fig. 7 are obtained for $\phi=80^{\circ}$.

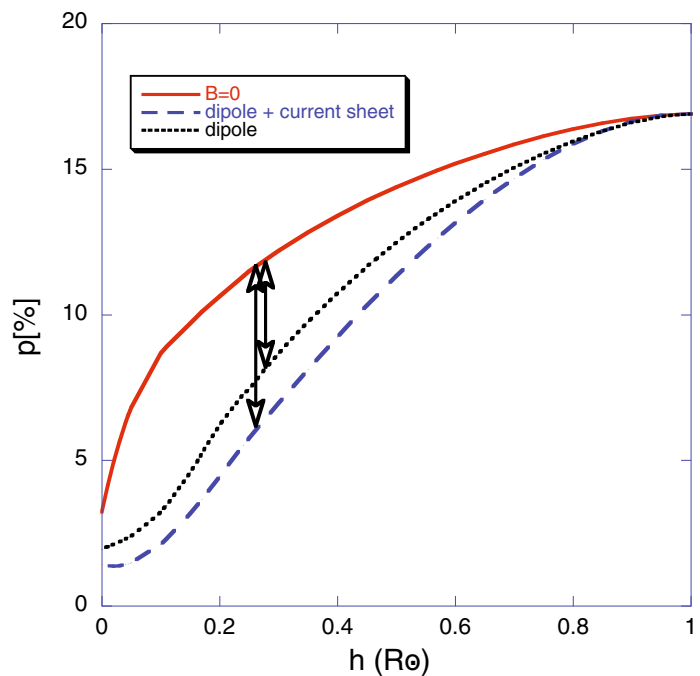

Fig. 7. Linear polarization degree versus the height from the solar surface $h$. We put together the results obtained in the zero-field case and these obtained for (1) a purely dipolar magnetic field (2) the sum of a dipolar field and a non-dipolar field associated to a current sheet with $\gamma=0.25$.

Our results show that $\Delta p \sim 5 \%$, i.e. well within the typical measurement sensitivities of a new generation of instruments such as LYOT (see Sect. 5). We point out that by using the UV SUMER spectrometer aboard SoHO, Raouafi et al. (1999) measured the linear polarization of the O VI $\lambda 1032$ line with a polarimetric precision equal to $1.7 \%$. We note in passing that such an accuracy is reached although SUMER was not initially designed to measure the polarization.

\section{Measurement of the linear polarization degree and its direction}

\subsection{Principle of the measurement}

Raouafi et al. (1999) used the rotation of the SUMER spectrometer to measure the linear polarization of the $\mathrm{D}_{2}$ component of the $\mathrm{O}$ VI $\lambda 1032$ line. They extracted the polarization of the $\mathrm{D}_{2}$ 
line from a ratio of the intensities of the non-polarizable $D_{1}$ line and of the $\mathrm{D}_{2}$ line (see the Fig. 3 of Raouafi et al. 1999). This technique was possible because the wavelengths of the two components $D_{1}$ and $D_{2}$ are sufficiently different to be resolved (1031.93 $\AA$ for the $\mathrm{D}_{2}$ line and $1037.62 \AA$ for the $\left.\mathrm{D}_{1}\right)$. However, the wavelengths of the $\mathrm{D}_{1}$ and $\mathrm{D}_{2}$ lines of the $\mathrm{L} \alpha$ line cannot be resolved since they are very close: in the vacuum $\lambda\left(D_{1}\right)=1215.668 \AA$ and $\lambda\left(D_{2}\right)=1215.674 \AA$. As a result, the technique presented by Fig. 3 of Raouafi et al. (1999) cannot be applied to measure the linear polarization of the $\mathrm{D}_{2}$ line of $\mathrm{L} \alpha$.

Using the so-called Poincaré representation ${ }^{4}$, one can demonstrate that the intensity observed when the instrument is placed at an arbitrary position referred by an angle $\beta$ around the LOS, is

$I(\beta)=\frac{1}{2}(Q \cos 2 \beta+U \sin 2 \beta+I)$.

In this expression the Stokes $V$ is assumed to be zero. The quantity $I$ denotes the unpolarized part of the intensity of the $\mathrm{D}_{1}$ and $\mathrm{D}_{2} \mathrm{~L} \alpha$ lines. $I(\beta)$ represents the "real" (polarized and unpolarized) observed intensity of the two resonance lines. In addition, taking into account that $\alpha_{0}$ corresponds to the direction of linear polarization (i.e. privileged direction of the electric field), Eq. (7) becomes

$I(\beta)=\frac{1}{2}\left(\sqrt{Q^{2}+U^{2}} \cos 2\left(\beta-\alpha_{0}\right)+I\right)$.

Note that $\cos 2 \alpha_{0}=\frac{Q}{\sqrt{Q^{2}+U^{2}}}$ and $\sin 2 \alpha_{0}=\frac{U}{\sqrt{Q^{2}+U^{2}}}$.

On the other hand, generally speaking, the linear polarization is defined as

$p=\frac{I_{\max }-I_{\min }}{I_{\max }+I_{\min }}$

where $I_{\max }$ and $I_{\min }$ are the maximum and minimum intensities. Using Eqs. (8) and (9), one finds that

$p=\frac{\sqrt{Q^{2}+U^{2}}}{I}$

and

$\frac{I(\beta)}{I}=\frac{1}{2}\left(p \cos 2\left(\beta-\alpha_{0}\right)+1\right)$.

In Eq. (11) we have three unknowns: $I, p$, and $\alpha_{0}$. Then, theoretically, the linear polarization state is fully obtained through only three measurements of the $I(\beta)$ which corresponds to three rotations of the polarizer-spectrometer. One takes for example $\beta=0, \frac{\pi}{4}$, and $\frac{\pi}{2}$, therefore:

$$
\begin{aligned}
\tan 2 \alpha_{0} & =\frac{2 I\left(\frac{\pi}{4}\right)-I}{I(0)-I\left(\frac{\pi}{2}\right)} \\
p & =\frac{I(0)-I\left(\frac{\pi}{2}\right)}{\cos 2 \alpha_{0} \times I}=\frac{2 I\left(\frac{\pi}{4}\right)-I}{\sin 2 \alpha_{0} \times I}
\end{aligned}
$$

where the intensity of the unpolarized light is given by:

$I=I(0)+I\left(\frac{\pi}{2}\right)$

Obviously, more than three measurements of $I(\beta)$ are welcome in order to increase the accuracy.

\footnotetext{
4 A suitable graphical representation of polarized light conceived by Henri Poincaré in 1892.
}

\subsection{The LYOT project}

The LYOT project is a $\mathrm{L} \alpha$ coronagraph combined with a $\mathrm{L} \alpha$ disk imager (see Vial et al. 2002; Millard et al. 2006; and Vial et al. 2008). In addition, it is planned to implement a simple polarizer system. The polarizing measurements will be performed by rotating the polarizer or the whole instrument to obtain the intensity of the L $\alpha$ light at different $\beta$ angles (previous section). The choice of the $\mathrm{L} \alpha$ line is well justified by its sensitivity to the coronal magnetic field (as demonstrated in this paper) and by the fact that in the corona the $\mathrm{L} \alpha$ emission is very intense. In fact, a high signal to noise ratio is needed since in the very low corona the anisotropy of the light is small, which in turn means that the polarization degree is small (smaller than 5\%, see Fig. 7). We note that no coronagraph observing as low as $1.15 R_{\odot}$ is envisaged beyond 2012 except for LYOT images which should be obtained with an excellent signal to noise ratio.

\section{Conclusion}

Measurement and interpretation of the scattering polarization of UV coronal lines provide a largely unexplored diagnostic of the coronal magnetic field. The greatest difficulty facing the UV coronal spectropolarimetry is that the polarization measurements integrate radiation along the LOS over structures with different properties but also that the observations of these lines are impossible from ground-based telescopes; they can only be observed with the help of high-sensitivity instruments flown on space missions.

We have performed a forward modeling of the coronal Hanle effect on the polarization of the $\mathrm{L} \alpha$ line generated by anisotropic scattering of chromospheric light. The main feature of this modeling consists in integrating the effect of the LOS. We show that the information about the coronal magnetic field is not lost through LOS integration. To confirm these results, we plan to work with different families of maps of magnetic fields and to add small scale magnetic perturbations. For instance, one can think of a set of active loops whose field determination could be compared with field extrapolations. One should however keep in mind that (1) a realistic thermodynamic model is required in order to integrate along the LOS and that (2) our modeling is limited to the case of optically thin plasmas in the $\mathrm{L} \alpha$ line.

Finally, we notice that it is suitable to combine measurements in $\mathrm{L} \alpha$ with measurements in polarized lines like the $\mathrm{Fe}$ XIV $\lambda 5303$ which have a different sensitivity to the magnetic field. An analysis of combined measurements should give more information data to constrain the magnetic field topology and strength (an example of the Hanle effect in a multi-line approach is given in Landi Degl'Innocenti 1982). It is also of interest to remark that the ground level ${ }^{2} \mathrm{~S}_{J}$ of $\mathrm{L} \alpha$ is non polarizable by radiation anisotropy, but that this is no longer true in the presence of hyperfine structure and if the depolarizing effect of the isotropic collisions is negligible. Because the sensitivity to the Hanle effect depends on the level life-time, the hyperfine polarization of such a long-lived level is much more vulnerable to very weak magnetic fields than the short-lived upper levels ${ }^{2} \mathrm{P}$. Consequently, one could distinguish a very small perturbation of the magnetic field (smaller than 1 Gauss) which corresponds for instance to a current sheet with a very small $\gamma$ and a background field of the order of 10 Gauss or larger. In particular, this could be the key to distinguish potential magnetic field structures from non potential ones. 
M. Derouich et al.: Hanle effect in the coronal $\mathrm{L} \alpha$ line

\section{References}

Auchère, F. 2005, ApJ, 622, 737

Blum, K. 1981, Density Matrix Theory and Applications (New York: Plenum Press)

Bommier, V., \& Sahal-Bréchot, S. 1982, Sol. Phys., 78, 157

Cranmer, S. R., Kohl, J. L., Noci, G., et al. 1999, ApJ, 511, 481

Cohen-Tannoudji, C., \& Kastler, A. 1966, in Progress in Optics, ed. E. Wolf, 5, 1

Fong, B., Low, B. C., \& Fan, Y. 2002, ApJ, 571, 987

Gabriel, A. H., Garton, W. R. S., Goldberg, L., et al. 1971, ApJ, 169, 595

Judge, P. G., Low, B. C., \& Casini, R. 2006, ApJ, 651, 1229

Landi Degl'Innocenti, E. 1982, Sol. Phys., 79, 291

Landi Degl'Innocenti, E. 1982, Sol. Phys., 91, 1

Landi Degl'Innocenti, E., \& Landolfi, M. 2004, Polarization in Spectral Lines (Dordrecht: Kluwer)

Low, B. C., Fong, B., \& Fan, Y. 2003, ApJ, 594, 1060
Millard, A. A., Lemaire, P., \& Vial, J. C., 2006, Proc. SPIE, 6266, 62662G

Manso Sainz, R., \& Trujillo Bueno, J. 2009, in Solar Polarization 5, ed. S. Berdyugina, K. N. Nagendra, \& R. Ramelli, ASP Conf. Ser., 405, 423

Omont A. 1977, Prog. Quantum Electronics, 5, 69

Raouafi, N.-E., Sahal-Bréchot, S., \& Lemaire, P. 1999, 9th European Meeting on Solar Physics, ed. A. Wilson, European Space Agency, ESA SP-448, 1205

Raouafi, N.-E., Sahal-Bréchot, S., \& Lemaire, P. 2002, A\&A, 396, 1019

Sahal-Bréchot, S. 1977, ApJ, 213, 887

Trujillo Bueno, J. 2009, in Magnetic Coupling between the Interior and the Atmosphere of the Sun, ed. S. S. Hasan, \& R. J. Rutten, Astrophysics and Space Science Proceedings (Springer-Verlag), in press [arXiv0903.4372T]

Trujillo Bueno, J., Landi degl'Innocenti, E., Casini, R., \& Marítnez Pillet, V. 2005, 39th ESLAB Symposium on Trends in Space Science and Cosmic Vision 2020, ed. F. Favata, J. Sanz-Forcada, A. Gimnez, \& B. Battrick, ESA SP-588, 203

Vial, J.-C., et al., 2002, The solar high resolution imager-coronograph LYOT mission, Astronomical telescopes and instrumentation, SPIE, 4853, 479

Vial, J.-C., Auchére, F., Chang, J., et al., 2008, Adv. Space Res., 41, 183 